\title{
A Variational Inequality from Pricing Convertible Bond
}

\author{
Huiwen Yan and Fahuai Yi \\ School of Mathematics, South China Normal University, Guangzhou 510631, China
}

Correspondence should be addressed to Fahuai Yi, fhyi@scnu.edu.cn

Received 30 December 2010; Accepted 11 February 2011

Academic Editor: Jin Liang

Copyright (C 2011 H. Yan and F. Yi. This is an open access article distributed under the Creative Commons Attribution License, which permits unrestricted use, distribution, and reproduction in any medium, provided the original work is properly cited.

The model of pricing American-style convertible bond is formulated as a zero-sum Dynkin game, which can be transformed into a parabolic variational inequality (PVI). The fundamental variable in this model is the stock price of the firm which issued the bond, and the differential operator in PVI is linear. The optimal call and conversion strategies correspond to the free boundaries of PVI. Some properties of the free boundaries are studied in this paper. We show that the bondholder should convert the bond if and only if the price of the stock is equal to a fixed value, and the firm should call the bond back if and only if the price is equal to a strictly decreasing function of time. Moreover, we prove that the free boundaries are smooth and bounded. Eventually we give some numerical results.

\section{Introduction}

Firms raise capital by issuing debt (bonds) and equity (shares of stock). The convertible bond is intermediate between these two instruments, which entitles its owner to receive coupons plus the return of principle at maturity. However, prior to maturity, the holder may convert the bond into the stock of the firm, surrendering it for a preset number of shares of stock. On the other hand, prior to maturity, the firm may call the bond forcing the bondholder to either surrender it to the firm for a previously agreed price or convert it into stock as before.

After issuing a convertible bond, the bondholder will find a proper time to exercise the conversion option in order to maximize the value of the bond, and the firm will choose its optimal time to exercise its call option to maximize the value of shareholder's equity. This situation was called "two-person" game (see $[1,2]$ ). Because the firm must pay coupons to the bondholder, it may call the bond if it can subsequently reissue a bond with a lower coupon rate. This happens as the firm's fortunes improve, then the risk of default has diminished and investors will accept a lower coupon rate on the firm's bonds. 
In [2] the authors assume that a firm's value is comprised of one equity and one convertible bond, the value of the issuing firm has constant volatility, the bond continuously pays coupons at a fixed rate, and the firm continuously pays dividends at a rate that is a fixed fraction of equity. Default occurs if the coupon payments cause the firm's value to fall to zero, in which case the bond has zero value. In their model, both the bond price and the stock price are functions of the underlying of the firm value. Because the stock price is the difference between firm value and bond price and dividends are paid proportionally to the stock price, a nonlinear differential equation was established for describing the bond price as a function of the firm value and time.

As we know, it is difficult to obtain the value of the firm. However, it is easier to get its stock price. So we choose the bond price $V(S, t)$ as a function of the stock price $S$ of the firm and time $t$ (see Chapter 36 in [3] or [4-7]).

In Section 2, we formulate the model and deduce that $V(S, t)=\gamma S$ in the domain $\{S \geq K / \gamma\}$ and $V(S, t)$ is governed by the following variational inequality in the domain $\{0 \leq S \leq K / \gamma\}$ :

$$
\begin{gathered}
-\partial_{t} V-\mathcal{L}_{0} V=c, \quad \text { if } V<K,(S, t) \in D_{T} \triangleq\left(0, \frac{K}{\gamma}\right) \times[0, T), \\
-\partial_{t} V-\mathcal{L}_{0} V \leq c, \quad \text { if } V=K,(S, t) \in D_{T}, \\
V\left(\frac{K}{\gamma}, t\right)=K, \quad 0 \leq t \leq T, \\
V(S, T)=\max \{L, \gamma S\}, \quad 0 \leq S \leq \frac{K}{\gamma}
\end{gathered}
$$

where $c, \gamma, K$, and $L$ are positive constants. $c$ is the coupon rate, $\gamma$ is the conversion ratio for converting the bond into the stock of the firm, $K$ is the call price of the firm, $L$ is the face value of the bond with $0<L \leq K$, and $\mathcal{L}_{0}$ is just B-S operator (see [8]),

$$
\mathcal{L}_{0} V=\frac{\sigma^{2}}{2} S^{2} \partial_{S S} V+(r-q) S \partial_{S} V-r V
$$

where $r, \sigma$, and $q$ are positive constants and represent the risk-free interest rate, the volatility, and the dividend rate of the firm stock, respectively. In this paper, we suppose that $c>r K$ and $r \geq q$. From a financial point of view, the assumption provides a possibility of calling the bond back from the firm (see Section 2 or [2]). Furthermore, we suppose that $L \leq K$. Otherwise, the firm should call the bond back before maturity and the value $L$ makes no sense (see Section 2). It is clear that $V=K$ is the unique solution if $L=K$. So we only consider the problem in the case of $L<K$.

Since (1.1) is a degenerate backward problem, we transform it into a familiar forward nondegenerate parabolic variational inequality problem; so letting

$$
u(x, t)=V(S, T-t), \quad x=\ln S-\ln K+\ln \gamma,
$$


we have that

$$
\begin{gathered}
\partial_{t} u-\mathcal{L} u=c, \quad \text { if } u<K, \quad(x, t) \in \Omega_{T} \triangleq(-\infty, 0) \times(0, T], \\
\partial_{t} u-\mathcal{L} u \leq c, \quad \text { if } u=K, \quad(x, t) \in \Omega_{T}, \\
u(0, t)=K, \quad 0 \leq t \leq T, \\
u(x, 0)=\max \left\{L, K e^{x}\right\}, \quad x \leq 0,
\end{gathered}
$$

where

$$
\mathcal{L} u=\frac{\sigma^{2}}{2} \partial_{x x} u+\left(r-q-\frac{\sigma^{2}}{2}\right) \partial_{x} u-r u
$$

There are many papers on the convertible bond, such as $[1,2,9]$. But as we know, there are seldom results on the properties of the free boundaries- the optimal call and conversion strategies in the existing literature. The main aim of this paper is to analyze some properties of the free boundaries.

The pricing model of the convertible bond without call is considered in [9], where there exist two domains: the continuation domain $\mathrm{CT}$ and the conversion domain $\mathrm{CV}$. The free boundary $S(t)$ between $\mathrm{CT}$ and $\mathrm{CV}$ means the optimal conversion strategy, which is dependent on the time $t$ and more than $K / \gamma$.

But in this model, their exist three domains: the continuation domain $\mathrm{CT}$, the callable domain $\mathrm{CL}$, and the conversion domain $\mathrm{CV}=\{x \geq 0\}$. The boundary between CV and CT $\cup$ CL is $x=0$, which means the call strategy. The free boundary $h(t)$ is the curve between CT and CL (see Figure 1), which means the optimal call strategy. And there exist $t_{0}, T_{0}$ such that

$$
0<t_{0}<T_{0} \triangleq \frac{1}{r} \ln \frac{c-r L}{c-r K}, \quad h(t) \in C\left[t_{0}, T_{0}\right) \cap C^{\infty}\left[t_{0}, T_{0}\right), \quad \lim _{t \rightarrow T_{0}^{-}} h(t)=-\infty,
$$

and $h(t)$ is strictly decreasing in $\left[t_{0}, T_{0}\right)$.

It means that the bondholder should convert the bond if and only if the stock price $S$ of the firm is no less than $K / \gamma$, whereas, in the model without call, the bondholder may not convert the bond even if $S>K / \gamma$. More precisely, the optimal conversion strategy $S(t)$ without call is more than that $K / \gamma$ in this paper (see [9] or Section 2). When the time to the expiry date is more than $T_{0}$, the firm should call the bond back if $S<K / \gamma$. Neither the bondholder nor the firm should exercise their option if the time to the expiry date is less than $t_{0}$ and $S<K e^{h(t)}$. Moreover, when the time to the maturity lies in $\left(t_{0}, T_{0}\right)$, the bondholder should call the bond back if $K e^{h(t)} \leq S<K / \gamma$.

In Section 2, we formulate and simplify the model. In Section 3, we will prove the existence and uniqueness of the strong solution of the parabolic variational inequality (1.4) and establish some estimations, which are important to analyze the property of the free boundary.

In Section 4, we show some behaviors of the free boundary $h(t)$, such as its starting point and monotonicity. Particularly, we obtain the regularity of the free boundary $h(t) \in$ $C^{0,1}\left[t_{0}, T_{0}\right) \cap C^{\infty}\left[t_{0}, T_{0}\right)$. As we know, the proof of the smoothness is trivial by the method 


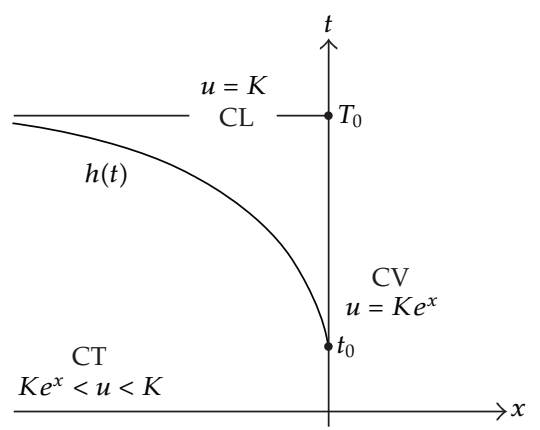

Figure 1: The free boundary $h(t)$.

in [10] if the difference between $u$ and the upper obstacle $K$ is decreasing with respect to $t$. But the proof is difficult if the condition is false (see [11-14]). In this problem, $\partial_{t}(u-K) \geq 0$, which does not match the condition. Moreover, $\partial_{x x} u(\ln L-\ln K, 0)=+\infty$, and the starting point $\left(0, t_{0}\right)$ of the free boundary $h(t)$ is not on the initial boundary, but the side boundary in this problem. Those make the proof of $h(t) \in C^{\infty}\left[t_{0}, T_{0}\right)$ more complicated. The key idea is to construct cone locally containing the local free boundary and prove $h(t) \in C^{0,1}\left[t_{0}, T_{0}\right)$; then the proof of $C^{\infty}\left[t_{0}, T_{0}\right)$ is trivial. Moreover, we show that there is a lower bound $h_{*}(t)$ of $h(t)$ and $h(t)$ converges to $-\infty$ as $t$ converges to $T_{0}^{-}$in Theorem 4.4 .

In the last section, we provide numerical result applying the binomial method.

\section{Formulation of the Model}

In this section, we derive the mathematical model of pricing the convertible bond.

The firm issues the convertible bond, and the bondholder buys the bond. The firm has an obligation to continuously serve the coupon payment to the bondholder at the rate of $c$. In the life time of the bond, the bondholder has the right to convert it into the firm's stock with the conversion factor $\gamma$ and obtains $\gamma S$ from the firm after converting, and the firm can call it back at a preset price of $K$. The bondholder's right is superior to the firm's, which means that the bondholder has the right to convert the bond, but the firm has no right to call it if both sides hope to exercise their rights at the same time. If neither the bondholder nor the firm exercises their right before maturity, the bondholder must sell the bond to the firm at a preset value $L$ or convert it into the firm's stock at expiry date. So, the bondholder receives $\max \{L, \gamma S\}$ from the firm at maturity. It is reasonable that both of them wish to maximize the values of their respective holdings.

Suppose that under the risk neutral probability space $(\Omega, \mathcal{F}, \mathbb{P})$; the stock price of the firm $S_{s}$ follows

$$
S_{s}^{t, S}=S+\int_{t}^{s}(r-q) S_{u}^{t, S} d u+\int_{t}^{s} \sigma S_{u}^{t, S} d W_{u}, \quad S \in[0,+\infty), s \in[t, T], t \in[0, T],
$$

where $r, q$, and $\sigma$ are positive constants, representing risk free interest rate, the dividend rate, and volatility of the stock, respectively. $W_{t}$ is a standard Brown motion on the probability space $(\Omega, \mathcal{F}, \mathbb{P})$. Usually, the dividend rate $q$ is smaller than the risk free interest rate $r$. So, we suppose that $q \leq r$. 
Denote by $\mathcal{F}_{t}$ the natural filtration generated by $W_{t}$ and augmented by all the $\mathbb{P}$-null sets in $\mathcal{F}$. Let $\mathcal{U}_{t, T}$ be the set of all $\mathcal{F}_{t}$-stopping times taking values in $[t, T]$.

The model can be expressed as a zero-sum Dynkin game. The payoff of the bondholder is

$$
\begin{aligned}
R(S, t ; \tau, \theta)= & \int_{t}^{\tau \wedge \theta} c e^{r t-r u} d u+e^{r t-r \tau} K I_{\{\tau<\theta\}}+e^{r t-r \theta} \gamma S_{\theta}^{t, S} I_{\{\theta \leq \tau, \theta<T\}} \\
& +e^{r t-r T} \max \left\{L, \gamma S_{T}^{t, S}\right\} I_{\{\tau \wedge \theta=T\}},
\end{aligned}
$$

where $\tau, \theta \in \mathfrak{U}_{t, T}$. The stopping time $\tau$ is the firm's strategy, and $\theta$ is the bondholder's strategy.

The bondholder chooses his strategy $\theta$ to maximize $R(S, t ; \tau, \theta)$; meanwhile, the firm chooses its strategy $\tau$ to minimize $R(S, t ; \tau, \theta)$.

Denote the upper value $\bar{V}$ and the lower value $\underline{V}$ as

$$
\begin{aligned}
& \underline{V}(S, t) \triangleq \text { ess sup ess } \operatorname{sinf}_{\theta \in \mathcal{U}_{t, T}} \mathbb{E}\left[R(S, t ; \tau, \theta) \mid \mathcal{F}_{t}\right], \\
& \bar{V}(S, t) \triangleq \text { ess } \inf _{\tau \in \mathcal{U}_{t, T}} \operatorname{ess} \sup _{\theta \in \mathcal{U}_{t, T}} \mathbb{E}\left[R(S, t ; \tau, \theta) \mid \mathcal{F}_{t}\right] .
\end{aligned}
$$

If $\bar{V}(S, t)=\underline{V}(S, t)$, then it is called the value of the Dynkin game and denoted as $V(S, t)$.

As we know, if the Dynkin game has a saddlepoint $\left(\tau^{*}, \theta^{*}\right) \in \mathfrak{U}_{t, T} \times \mathfrak{U}_{t, T}$, that is,

$$
\mathbb{E}\left[R\left(S, t ; \tau^{*}, \theta\right) \mid \mathcal{F}_{t}\right] \leq \mathbb{E}\left[R\left(S, t ; \tau^{*}, \theta^{*}\right) \mid \mathcal{F}_{t}\right] \leq \mathbb{E}\left[R\left(S, t ; \tau, \theta^{*}\right) \mid \mathcal{F}_{t}\right], \quad \forall \tau, \theta \in \mathcal{U}_{t, T},
$$

then the value of the Dynkin game exists and

$$
V(S, t)=\mathbb{E}\left[R\left(S, t ; \tau^{*}, \theta^{*}\right) \mid \mathcal{F}_{t}\right] .
$$

If $S \geq K / \gamma$, then we deduce that, for any $\tau, \theta \in \mathfrak{U}_{t, T}$,

$$
\begin{gathered}
\mathbb{E}\left[R(S, t ; \tau, t) \mid \mathcal{F}_{t}\right]=\gamma S I_{\{t<T\}}+\max \{L, \gamma S\} I_{\{t=T\}}=\mathbb{E}\left[R(S, t ; t, t) \mid \mathcal{F}_{t}\right], \\
\mathbb{E}\left[R(S, t ; t, \theta) \mid \mathscr{F}_{t}\right]=\mathbb{E}\left[K I_{\{t<\theta\}}+\gamma S I_{\{\theta=t\}} \mid \mathcal{F}_{t}\right] I_{\{t<T\}}+\max \{L, \gamma S\} I_{\{t=T\}} \leq \mathbb{E}\left[R(S, t ; t, t) \mid \mathcal{F}_{t}\right] .
\end{gathered}
$$

So, in this case, $(t, t)$ is a saddlepoint, and the value of the Dynkin game is

$$
V(S, t)=\gamma S I_{\{t<T\}}+\max \{L, \gamma S\} I_{\{t=T\}}, \quad \forall S \geq \frac{K}{\gamma}
$$


In the case of $0<S<K / \gamma$, applying the standard method in [15], we see that the strong solution of the following variational inequality is the value of the Dynkin game:

$$
\begin{gathered}
-\partial_{t} V-\mathcal{L}_{0} V=c, \quad \text { if } \gamma S<V<K,(S, t) \in D_{T}, \\
-\partial_{t} V-\mathcal{L}_{0} V \geq c, \quad \text { if } V=\gamma S,(S, t) \in D_{T}, \\
-\partial_{t} V-\mathcal{L}_{0} V \leq c, \quad \text { if } V=K,(S, t) \in D_{T}, \\
V\left(\frac{K}{r}, t\right)=K, \quad 0 \leq t \leq T, \\
V(S, T)=\max \{L, \gamma S\}, \quad 0 \leq S \leq \frac{K}{\gamma} .
\end{gathered}
$$

If $L>K$, then the firm is bound to call the bond back before the maturity because the firm pays $K$ after calling, but more than $L$ without calling. In this case, the value $L$ makes no sense. So, we suppose that $L \leq K$.

If $c \leq r K$, then the firm is bound to abandon its call right. From a financial point of view, the firm would pay $K$ to the bondholder at time $t$ after calling the bond, whereas, if the firm does not call in the time interval $[t, t+d t]$, then he would pay the coupon payment $c d t$ and at most $K$ of the face value of the convertible bond at time $t+d t$. So, the discounted value of the bond without call is at most $K+c d t-r K d t \leq K$. Hence, the firm should not call the bond back at time $t$.

From a stochastic point of view, we can denote a stopping time

$$
\tau_{1}=\inf \left\{t \leq u \leq T: \gamma S_{u}^{t, S} \geq K\right\}
$$

If $t<T, 0<S<K / \gamma$, then $\mathbb{P}\left(\tau_{1}>t\right)=1$, and, for any $\theta \in \mathfrak{U}_{t, T}$, we have

$$
\begin{aligned}
R\left(S, t ; \tau_{1}, \theta\right)= & \frac{c}{r}+I_{\left\{\tau_{1}<\theta\right\}} e^{r t-r \tau_{1}}\left(K-\frac{c}{r}\right)+I_{\left\{\theta \leq \tau_{1}, \theta<T\right\}} e^{r t-r \theta}\left(r S_{\theta}^{t, S}-\frac{c}{r}\right) \\
& +I_{\left\{\tau_{1} \wedge \theta=T\right\}} e^{r t-r T}\left(\max \left\{L, \gamma S_{T}^{t, S}\right\}-\frac{c}{r}\right) \\
\leq & \frac{c}{r}+e^{r t-r\left(\tau_{1} \wedge \theta \wedge T\right)}\left(K-\frac{c}{r}\right)=K \leq R(S, t ; t, \theta) \quad \text { a.s. in } \Omega .
\end{aligned}
$$

Moreover, $\mathbb{P}\left(R\left(S, t ; \tau_{1}, \theta\right)<R(S, t ; t, \theta)\right)=1$. So, for any $\tau, \theta \in \mathcal{U}_{t, T}$ such that $\mathbb{P}(\tau=t)>0$, it is clear that in the domain $\{t<T, 0<S<K / \gamma\}$

$$
\mathbb{E}\left[R(S, t ; \tau, \theta) \mid \mathscr{F}_{t}\right]>\mathbb{E}\left[R\left(S, t ; \tau I_{\{\tau>t\}}+\tau_{1} I_{\{\tau=t\}}, \theta\right) \mid \mathcal{F}_{t}\right]
$$

which means that $\tau$ is not the optimal call strategy, and the firm should not call in the domain $\{t<T, 0<S<K / \gamma\}$. 
From a variational inequality point of view, since

$$
-\partial_{t} K-\mathcal{L}_{0} K=r K>c,
$$

provided that $c<r K$, which contradicts with the third inequality in (2.8), so, if $c<r K$, then $V \neq K$ in the domain $\{t<T, 0<S<K \gamma\}$.

To remain the call strategy, we suppose that $c>r K$. We will consider the other case in another paper because the two problems are fully different.

Since we suppose that $c>r K$ and $r \geq q$, then

$$
-\partial_{t}(r S)-\left\llcorner_{0}(r S)=q r S \leq r K<c .\right.
$$

Hence, $\{V=\gamma S\}$ is empty in problem (2.8). So, problem (2.8) is reduced into problem (1.1). The model of pricing the bond without call is an optimal stopping problem

$$
\begin{gathered}
U(S, t) \triangleq \sup _{\theta \in \mathcal{U}_{t, T}} \mathbb{E}\left[Q(S, t ; \theta) \mid \mathcal{F}_{t}\right], \\
Q(S, t ; \theta)=\int_{t}^{\theta} c e^{r t-r u} d u+e^{r t-r \theta} \gamma S_{\theta}^{t, S} I_{\{\theta<T\}}+e^{r t-r T} \max \left\{L, \gamma S_{T}^{t, S}\right\} I_{\{\theta=T\}} .
\end{gathered}
$$

It is clear that

$$
U(S, t)=\operatorname{ess} \sup _{\theta \in \mathcal{U}_{t, T}} \mathbb{E}\left[R(S, t ; T, \theta) \mid \mathcal{F}_{t}\right] \geq V(S, t)
$$

Since $U, V \geq r S$, then

$$
\{U=r S\} \subset\{V=r S\}, \quad C V^{*} \subset C V,
$$

where $\mathrm{CV}^{*}$ is the conversion domain in the model without call and $\mathrm{CV}$ is that in this paper.

\section{The Existence and Uniqueness of $W_{p, l o c}^{2,1}$ Solution of Problem (1.4)}

Since problem (1.4) lies in the unbounded domain $\Omega_{T}$, we need the following problem in the bounded domain $\Omega_{T}^{n} \triangleq(-n, 0) \times(0, T]$ to approximate to problem (1.4):

$$
\begin{gathered}
\partial_{t} u_{n}-\mathcal{L} u_{n}=c, \quad \text { if } u_{n}<K,(x, t) \in \Omega_{T}^{n}, \\
\partial_{t} u_{n}-\mathcal{L} u_{n} \leq c, \quad \text { if } u_{n}=K, \quad(x, t) \in \Omega_{T}^{n}, \\
u_{n}(-n, t)=L, \quad u_{n}(0, t)=K, \quad 0 \leq t \leq T, \\
u_{n}(x, 0)=\max \left\{L, K e^{x}\right\}, \quad-n \leq x \leq 0,
\end{gathered}
$$

where $n \in I N_{+}$and $n>\ln K-\ln L$. 
Following the idea in $[10,16]$, we construct a penalty function $\beta_{\varepsilon}(s)$ (see Figure 2), which satisfies

$$
\begin{gathered}
\varepsilon>0 \text { and small enough, } \quad \beta_{\varepsilon}(s) \in C^{\infty}(-\infty,+\infty), \\
\beta_{\varepsilon}(s)=0, \quad \text { if } s \leq-\varepsilon, \quad \beta_{\varepsilon}(0)=C_{0} \triangleq c-r K>0, \\
\beta_{\varepsilon}(s) \geq 0, \quad \beta_{\varepsilon}^{\prime}(s) \geq 0, \quad \beta_{\varepsilon}^{\prime \prime}(s) \geq 0 \\
\lim _{\varepsilon \rightarrow 0} \beta_{\varepsilon}(s)= \begin{cases}0, & s<0 \\
+\infty, & s>0 .\end{cases}
\end{gathered}
$$

Consider the following penalty problem of (3.1):

$$
\begin{gathered}
\partial_{t} u_{\varepsilon, n}-\mathcal{L} u_{\varepsilon, n}+\beta_{\varepsilon}\left(u_{\varepsilon, n}-K\right)=c, \quad \text { in } \Omega_{T}^{n} \\
u_{\varepsilon, n}(-n, t)=L, \quad u_{\varepsilon, n}(0, t)=K, \quad 0 \leq t \leq T, \\
u_{\varepsilon, n}(x, 0)=\pi_{\varepsilon}\left(K e^{x}-L\right)+L, \quad-n \leq x \leq 0,
\end{gathered}
$$

where $\pi_{\varepsilon}(s)$ is a smoothing function because the initial value $\max \left\{L, K e^{x}\right\}$ is not smooth. It satisfies (see Figure 3)

$$
\begin{gathered}
\pi_{\varepsilon}(s)= \begin{cases}s, & s \geq \varepsilon, \\
0, & s \leq-\varepsilon,\end{cases} \\
\pi_{\varepsilon}(s) \in C^{\infty}(I R), \quad \pi_{\varepsilon}(s) \geq s, \quad 0 \leq \pi_{\varepsilon}^{\prime}(s) \leq 1, \quad \pi_{\varepsilon}^{\prime \prime}(s) \geq 0, \quad \lim _{\varepsilon \rightarrow 0^{+}} \pi_{\varepsilon}(s)=s^{+} .
\end{gathered}
$$

Lemma 3.1. For any fixed $\varepsilon>0$, problem (3.3) has a unique solution $u_{\varepsilon, n} \in W_{p}^{2,1}\left(\Omega_{T}^{n}\right) \cap C\left(\overline{\Omega_{T}^{n}}\right)$ for any $1<p<+\infty$ and

$$
\begin{gathered}
\max \left\{L, K e^{x}\right\} \leq u_{\varepsilon, n} \leq K \quad \text { in } \overline{\Omega_{T}^{n}} \\
\partial_{x} u_{\varepsilon, n} \geq 0 \quad \text { in } \overline{\Omega_{T}^{n}}
\end{gathered}
$$

Proof. We apply the Schauder fixed point theorem [17] to prove the existence of nonlinear problem (3.3).

Denote $B=C\left(\overline{\Omega_{T}^{n}}\right)$ and $D=\{w \in B: w \leq c / r\}$. Then $D$ is a closed convex set in $B$. Defining a mapping $\mathcal{F}$ by $\mathcal{F}(w)=u_{\varepsilon, n}$ is the solution of the following linear problem:

$$
\begin{gathered}
\partial_{t} u_{\varepsilon, n}-\mathcal{L} u_{\varepsilon, n}+\beta_{\varepsilon}(w-K)=c \quad \text { in } \Omega_{T}^{n}, \\
u_{\varepsilon, n}(-n, t)=L, \quad u_{\varepsilon, n}(0, t)=K, \quad 0 \leq t \leq T, \\
u_{\varepsilon, n}(x, 0)=\pi_{\varepsilon}\left(K e^{x}-L\right)+L, \quad-n \leq x \leq 0 .
\end{gathered}
$$




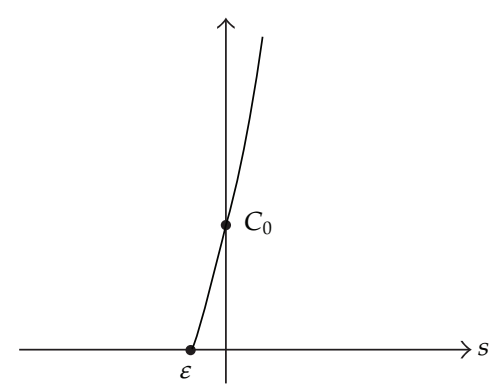

Figure 2: The function $\beta_{\varepsilon}$.

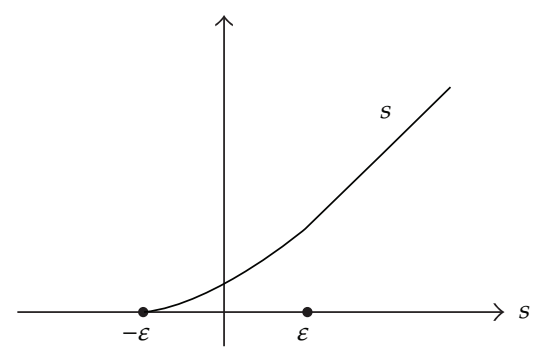

Figure 3: The function $\pi_{\varepsilon}$.

Furthermore, we can compute

$$
\begin{gathered}
\partial_{t}\left(\frac{c}{r}\right)-\mathcal{L}\left(\frac{c}{r}\right)+\beta_{\varepsilon}(w-K)=r \frac{c}{r}+\beta_{\varepsilon}(w-K) \geq c, \\
\frac{c}{r}>K \geq u_{\varepsilon, n} \quad \text { on } \partial_{p} \Omega_{T}^{n},
\end{gathered}
$$

where $\partial_{p} \Omega_{T}^{n}$ is the parabolic boundary of $\Omega_{T}^{n}$. Thus $c / r$ is a supersolution of the problem (3.7), and $u_{\varepsilon, n} \leq c / r$. Hence $\mathcal{F}(D) \subset D$. On the other hand,

$$
0 \leq \beta_{\varepsilon}(w-K) \leq \beta_{\varepsilon}\left(\frac{c}{r}-K\right)
$$

which is bounded for fixed $\varepsilon>0$. So, it is not difficult to prove that $\overline{\mathcal{F ( D )}}$ is compact in $B$ and $F$ is continuous. Owing to the Schauder fixed point theorem, we know that problem (3.3) has a solution $u_{\varepsilon, n} \in W_{p}^{2,1}\left(\Omega_{T}^{n}\right)$. The proof of the uniqueness follows by the comparison principle. Here, we omit the details.

Now, we prove (3.5). Since

$$
\begin{gathered}
\partial_{t} K-\mathcal{L} K+\beta_{\varepsilon}(K-K)=r K+\beta_{\varepsilon}(0)=c, \\
K \geq u_{\varepsilon, n} \quad \text { on } \partial_{p} \Omega_{T}^{n} .
\end{gathered}
$$


Therefore, $K$ is a supersolution of problem (3.3), and $u_{\varepsilon, n} \leq K$ in $\overline{\Omega_{T}^{n}}$. Moreover,

$$
\begin{gathered}
\partial_{t}\left(K e^{x}\right)-\mathcal{L}\left(K e^{x}\right)+\beta_{\varepsilon}\left(K e^{x}-K\right)=q K e^{x}+\beta_{\varepsilon}\left(K e^{x}-K\right) \leq q K e^{x}+\beta_{\varepsilon}(0)=q K e^{x}+c-r K \leq c, \\
\left.K e^{x}\right|_{x=-n}=K e^{-n} \leq L=u_{\varepsilon, n}(-n, t),\left.\quad K e^{x}\right|_{x=0}=K=u_{\varepsilon, n}(0, t), \\
K e^{x} \leq \max \left\{K e^{x}, L\right\} \leq \pi_{\varepsilon}\left(K e^{x}-L\right)+L=u_{\varepsilon, n}(x, 0) .
\end{gathered}
$$

Hence, $K e^{x}$ is a subsolution of problem (3.3). On the other hand,

$$
\begin{gathered}
\partial_{t} L-\mathcal{L} L+\beta_{\varepsilon}(L-K)=r L+\beta_{\varepsilon}(L-K) \leq r L+\beta_{\varepsilon}(0) \leq c, \\
L=u_{\varepsilon, n}(-n, t), \quad L<K=u_{\varepsilon, n}(0, t), \\
L \leq \max \left\{K e^{x}, L\right\} \leq \pi_{\varepsilon}\left(K e^{x}-L\right)+L=u_{\varepsilon, n}(x, 0) .
\end{gathered}
$$

Thus, $L$ is a subsolution of problem (3.3) as well, and we deduce $u_{\varepsilon, n} \geq \max \left\{K e^{x}, L\right\}$.

In the following, we prove (3.6).

Indeed, $u_{\varepsilon, n} \leq K$ and $u_{\varepsilon, n}(0, t)=K$ imply that $\partial_{x} u_{\varepsilon, n}(0, t) \geq 0$. Furthermore, $u_{\varepsilon, n} \geq L$ and $u_{\varepsilon, n}(-n, t)=L$ that imply $\partial_{x} u_{\varepsilon, n}(-n, t) \geq 0$. Differentiating (3.3) with respect to $x$ and denoting $W=\partial_{x} u_{\varepsilon, n}$, we obtain

$$
\begin{gathered}
\partial_{t} W-\mathcal{L} W+\beta_{\varepsilon}^{\prime}\left(u_{\varepsilon, n}-K\right) W=0 \quad \text { in } \Omega_{T}^{n} \\
W(-n, t) \geq 0, \quad W(0, t) \geq 0, \quad 0 \leq t \leq T, \\
W(x, 0)=\pi_{\varepsilon}^{\prime}\left(K e^{x}-L\right) K e^{x} \geq 0, \quad-n \leq x \leq 0 .
\end{gathered}
$$

Then the comparison principle implies (3.6).

Theorem 3.2. For any fixed $n \in I N, n>\ln K-\ln L$, problem (3.1) admits a unique solution $u_{n} \in$ $C\left(\overline{\Omega_{T}^{n}}\right) \cap W_{p}^{2,1}\left(\Omega_{T}^{n} \backslash B_{\delta}\left(P_{0}\right)\right)$ for any $1<p<+\infty, 0<\delta<n$, where $P_{0}=(-\ln K+\ln L, 0)$, $B_{\delta}\left(P_{0}\right)=\left\{(x, t):(x+\ln K-\ln L)^{2}+t^{2} \leq \delta^{2}\right\}$. Moreover, if $n$ is large enough, one has that

$$
\begin{gathered}
\max \left\{L, K e^{x}\right\} \leq u_{n} \leq K \quad \text { in } \Omega_{T}^{n}, \\
\partial_{x} u_{n} \geq 0 \quad \text { in } \Omega_{T}^{n}, \\
\partial_{t} u_{n} \geq 0 \quad \text { a.e. in } \Omega_{T}^{n} .
\end{gathered}
$$

Proof. From (3.5) and the properties of $\beta_{\varepsilon}(s)$, we have that

$$
0 \leq \beta_{\varepsilon}\left(u_{\varepsilon, n}-K\right) \leq \beta_{\varepsilon}(0)=c-r K \text {. }
$$

By $W_{p}^{2,1}$ and $C^{\alpha, \alpha / 2}(0<\alpha<1)$ estimates of the parabolic problem [18], we conclude that

$$
\left\|u_{\varepsilon, n}\right\|_{W_{p}^{2,1}\left(\Omega_{T}^{n} \backslash B_{\delta}\left(P_{0}\right)\right)}+\left\|u_{\varepsilon, n}\right\|_{C^{\alpha, \alpha / 2}\left(\overline{\Omega_{T}^{n}}\right)} \leq C,
$$


where $C$ is independent of $\varepsilon$. It implies that there exists a $u_{n} \in W_{p}^{2,1}\left(\Omega_{T}^{n} \backslash B_{\delta}\left(P_{0}\right)\right) \cap C\left(\overline{\Omega_{T}^{n}}\right)$ and a subsequence of $\left\{u_{\varepsilon, n}\right\}$ (still denoted by $\left\{u_{\varepsilon, n}\right\}$ ), such that as $\varepsilon \rightarrow 0^{+}$,

$$
u_{\varepsilon, n} \rightarrow u_{n} \quad \text { in } W_{p}^{2,1}\left(\Omega_{T}^{n} \backslash B_{\delta}\left(P_{0}\right)\right) \text { weakly, } \quad u_{\varepsilon, n} \longrightarrow u_{n} \quad \text { in } C\left(\overline{\Omega_{T}^{n}}\right) .
$$

Employing the method in [16] or [19], it is not difficult to derive that $u_{n}$ is the solution of problem (3.1). And (3.14), (3.15) are the consequence of (3.5), (3.6) as $\varepsilon \rightarrow 0^{+}$.

In the following, we will prove (3.16). For any small $\delta>0, w(x, t) \triangleq u_{n}(x, t+\delta)$ satisfies, by (3.1),

$$
\begin{gathered}
\partial_{t} w-\mathcal{L} w=c, \quad \text { if } w<K,(x, t) \in(-n, 0) \times(0, T-\delta], \\
\partial_{t} w-\mathcal{L} w \leq c, \quad \text { if } w=K,(x, t) \in(-n, 0) \times(0, T-\delta], \\
w(-n, t)=L=u_{n}(-n, t), \quad w(0, \delta)=K=u_{n}(0, t), \quad 0 \leq t \leq T-\delta, \\
w(x, 0)=u_{n}(x, \delta) \geq \max \left\{L, K e^{x}\right\}=u_{n}(x, 0), \quad-n \leq x \leq 0 .
\end{gathered}
$$

Applying the comparison principle with respect to the initial value of the variational inequality (see [16]), we obtain

$$
u_{n}(x, t+\delta)=w(x, t) \geq u_{n}(x, t), \quad(x, t) \in(-n, 0) \times(0, T-\delta]
$$

Thus (3.16) follows.

At last, we prove the uniqueness of the solution. Suppose that $u_{n}^{1}$ and $u_{n}^{2}$ are two $W_{p, \text { loc }}^{2,1}\left(\Omega_{T}^{n}\right) \cap C\left(\overline{\Omega_{T}^{n}}\right)$ solutions to problem (3.1), and denote

$$
\mathcal{N} \triangleq\left\{(x, t) \in \Omega_{T}^{n}: u_{n}^{1}(x, t)<u_{n}^{2}(x, t)\right\} .
$$

Assume that $\mathcal{N}$ is not empty, and then, in the domain $\mathcal{N}$,

$$
u_{n}^{1}(x, t)<u_{n}^{2}(x, t) \leq K, \quad \partial_{t} u_{n}^{1}-\mathcal{L} u_{n}^{1}=c, \quad \partial_{t}\left(u_{n}^{1}-u_{n}^{2}\right)-\mathcal{L}\left(u_{n}^{1}-u_{n}^{2}\right) \geq 0 .
$$

Denoting $W=u_{n}^{1}-u_{n}^{2}$, we have that

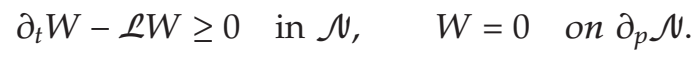

Applying the A-B-P maximum principle (see [20]), we have that $W \geq 0$ in $\mathcal{N}$, which contradicts the definition of $\mathcal{N}$. 
Theorem 3.3. Problem (1.4) has a unique solution $u \in C\left(\bar{\Omega}_{T}\right) \cap W_{p}^{2,1}\left(\Omega_{T}^{R} \backslash B_{\delta}\left(P_{0}\right)\right)$ for any $1<p<$ $+\infty, R>0$, and $\delta>0$. And $\partial_{x} u \in C\left(\bar{\Omega}_{T} \backslash B_{\delta}\left(P_{0}\right)\right)$. Moreover,

$$
\begin{gathered}
\max \left\{L, K e^{x}\right\} \leq u \leq K \quad \text { in } \bar{\Omega}_{T}, \\
\partial_{x} u \geq 0 \quad \text { a.e. in } \bar{\Omega}_{T}, \\
\partial_{t} u \geq 0 \quad \text { a.e. in } \Omega_{T} .
\end{gathered}
$$

Proof. Rewrite Problem (3.1) as follows:

$$
\begin{gathered}
\partial_{t} u_{n}-\mathcal{L} u_{n}=f(x, t), \quad(x, t) \in \Omega_{T^{\prime}}^{n} \\
u_{n}(-n, t)=L, \quad u_{n}(0, t)=K, \quad 0 \leq t \leq T, \\
u_{n}(x, 0)=\max \left\{L, K e^{x}\right\}, \quad-n \leq x \leq 0,
\end{gathered}
$$

where $u_{n} \in W_{p}^{2,1}\left(\Omega_{T}^{n} \backslash B_{\delta}\left(P_{0}\right)\right)$ implies that $f(x, t) \in L_{\mathrm{loc}}^{p}\left(\Omega_{T}^{n}\right)$ and

$$
f(x, t)=c I_{\left\{u_{n}<K\right\}}+r K I_{\left\{u_{n}=K\right\}},
$$

where $I_{A}$ denotes the indicator function of the set $A$.

Hence, for any fixed $R>\delta>0$, if $n>R$, combining (3.14), we have the following $W_{p}^{2,1}$ and $C^{\alpha, \alpha / 2}$ uniform estimates [18]:

$$
\left\|u_{n}\right\|_{W_{p}^{2,1}\left(\Omega_{T}^{R} \backslash B_{\delta}\left(P_{0}\right)\right)} \leq C_{R, \delta}, \quad\left\|u_{n}\right\|_{C^{\alpha, \alpha / 2}\left(\bar{\Omega}_{T}^{R}\right)} \leq C_{R}
$$

here $C_{R, \delta}$ depends on $R$ and $\delta, C_{R}$ depends on $R$, but they are independent of $n$. Then, we have that there is a $u \in W_{p, \text { loc }}^{2,1}\left(\Omega_{T}\right) \cap C(\bar{\Omega})_{T}$ and a subsequence of $\left\{u_{n}\right\}$ (still denoted by $\left\{u_{n}\right\}$ ), such that for any $R>\delta>0, p>1$,

$$
u_{n} \rightarrow u \text { in } W_{p}^{2,1}\left(\Omega_{T}^{R} \backslash B_{\delta}\left(P_{0}\right)\right) \text { weakly as } n \longrightarrow+\infty \text {. }
$$

Moreover, (3.30) and imbedding theorem imply that

$$
u_{n} \longrightarrow u \quad \text { in } C\left(\bar{\Omega}_{T}^{R}\right), \quad \partial_{x} u_{n} \longrightarrow \partial_{x} u \quad \text { in } C\left(\bar{\Omega}_{T}^{R} \backslash B_{\delta}\left(P_{0}\right)\right) \quad \text { as } n \longrightarrow+\infty
$$

It is not difficult to deduce that $u$ is the solution of problem (1.4). Furthermore, (3.32) implies that $\partial_{x} u \in C\left(\bar{\Omega}_{T} \backslash B_{\delta}\left(P_{0}\right)\right)$. And (3.25)-(3.27) are the consequence of (3.14)-(3.16). The proof of the uniqueness is similar to the proof in Theorem 3.2. 


\section{Behaviors of the Free Boundary}

Denote

$$
\begin{gathered}
\mathrm{CT}=\{(x, t): u(x, t)<K\} \quad \text { (continuation region), } \\
\mathrm{CL}=\{(x, t): u(x, t)=K\} \quad \text { (callable region). }
\end{gathered}
$$

Thanks to (3.26), we can define the free boundary $h(t)$ of problem (1.4), at which it is optimal for the firm to call the bond, where

$$
h(t)=\inf \{x \leq 0: u(x, t)=K\}, \quad 0<t \leq T
$$

(see Figure 1). It is clear that

$$
\mathrm{CT}=\{x<h(t)\}, \quad \mathrm{CL}=\{h(t) \leq x<0\} .
$$

Theorem 4.1. Denote $T_{0}=(1 / r) \ln ((c-r L) /(c-r K))$. If $t \geq T_{0}$, then $u(x, t) \equiv K$, which means that

$$
C T \subset\left\{0<t<T_{0}, x<0\right\}, \quad C L \supset\left\{t \geq T_{0}, x<0\right\}, \quad h(t)=-\infty \quad \text { for any } t \geq T_{0} .
$$

Proof. Define

$$
w(x, t)= \begin{cases}\frac{c}{r}-\left(\frac{c}{r}-L\right) e^{-r t}, & 0 \leq t \leq T_{0} \\ K, & T_{0} \leq t \leq T\end{cases}
$$

We claim that $w(x, t)$ possess the following four properties.

(i) $w \in W_{p, \text { loc }}^{2,1}\left(\Omega_{T}\right) \cap C\left(\overline{\Omega_{T}}\right)$,

(ii) $w \leq K$, for all $(x, t) \in \overline{\Omega_{T}}$,

(iii) $w(x, 0)=L \leq \max \left\{L, K e^{x}\right\}=u(x, 0)$, for all $x \in(-\infty, 0]$,

(iv) $\partial_{t} w-\mathcal{L} w \leq c$, a.e. in $\Omega_{T}$.

In fact, from the definition of $T_{0}$, we have that

$$
w\left(x, T_{0}\right)=\frac{c}{r}-\left(\frac{c}{r}-L\right) \exp \left\{-r \frac{1}{r} \ln \frac{c-r L}{c-r K}\right\}=K,
$$

then property (i) is obvious.

Moreover, if $0<t \leq T_{0}$, then we deduce

$$
\partial_{t} w(x, t)=r\left(\frac{c}{r}-L\right) e^{-r t} \geq 0 .
$$


Combining $w\left(x, T_{0}\right)=K$, we have property (ii). It is easy to check property (iii) from the definition of $w$. Next, we manifest property (iv) according to the following two cases. In the case of $0<t \leq T_{0}$,

$$
\partial_{t} w-\mathcal{L} w=r\left(\frac{c}{r}-L\right) e^{-r t}+r\left[\frac{c}{r}-\left(\frac{c}{r}-L\right) e^{-r t}\right]=c .
$$

In the other case of $T_{0}<t \leq T$,

$$
\partial_{t} w-\perp w=r K<c
$$

So, we testify properties (i)-(iv). In the following, we utilize the properties to prove $w \leq u$.

Otherwise, $N=\{w>u\}$ is nonempty; then we have that

$$
u(x, t)<w(x, t) \leq K, \quad \partial_{t} u-\mathcal{L} u=c, \quad \partial_{t}(u-w)-\mathcal{L}(u-w) \geq 0, \quad \text { in } \mathcal{N} .
$$

Moreover, $u-w \geq 0$ on the parabolic boundary of $\mathcal{N}$. According to the A-B-P maximum principle (see [20]), we have that

$$
u-w \geq 0 \text { in } \mathcal{N}
$$

which contradicts the definition of $\Omega$. So, we achieve that $w \leq u$.

Combining $w(x, t)=K$ for any $t \geq T_{0}$, it is clear that

$$
K=w(x, t) \leq u(x, t) \leq K, \quad \text { for any } t \geq T_{0},
$$

which means that CT $\subset\left\{0<t<T_{0}, x<0\right\}, \mathrm{CL} \supset\left\{t \geq T_{0}, x<0\right\}$, and $h(t)=-\infty$ for any $t \geq T_{0}$

Theorem 4.2. The free boundary $h(t)$ is decreasing in the interval $\left(0, T_{0}\right)$. Moreover, $h(0) \triangleq$ $\lim _{t \rightarrow 0^{+}} h(t)=0$. And $h(t) \in C\left[0, T_{0}\right)$.

Proof. (3.26) and (3.27) imply that

$$
\partial_{x}(u-K) \geq 0, \quad \partial_{t}(u-K) \geq 0 \quad \text { a.e. in } \Omega_{T} .
$$

Hence, for any unit vector $n=\left(n_{1}, n_{2}\right)$ satisfying $n_{1}, n_{2}>0$, the directional derivative of function $u-K$ along $n$ admits

$$
\partial_{n}(u-K) \geq 0 \text { a.e. in } \Omega_{T},
$$

that is, $u-K$ is increasing along the director $n$. Combining the condition $u-K \leq 0$ in $\Omega_{T}$, we know that $x=h(t)$ is monotonically decreasing. Hence, $\lim _{t \rightarrow 0^{+}} h(t)$ exists, and we can define

$$
h(0)=\lim _{t \rightarrow 0^{+}} h(t) .
$$


Since $u(0, t)=K$, so $h(0) \leq 0$. On the other hand, if $h(0)<0$, then

$$
u(x, t)=K, \quad \forall(x, t) \in(h(0), 0) \times(0, T], \quad u(x, 0)=\max \left\{L, K e^{x}\right\}<K, \quad \forall x \in(h(0), 0) .
$$

It is impossible because $u$ is continuous on $\bar{\Omega}_{T}$.

In the following, we prove that $h(t)$ is continuous in $\left(0, T_{0}\right)$. If it is false, then there exists $x_{1}<x_{2}<0,0<t_{1}<T_{0}$ such that (see Figure 4)

$$
\lim _{t \rightarrow t_{1}^{-}} h(t)=x_{1}, \quad \lim _{t \rightarrow t_{1}^{+}} h(t)=x_{2}
$$

Moreover,

$$
\partial_{t} u-\mathcal{L} u=c \quad \text { in } \mathcal{M} \triangleq\left\{(x, t): x_{2}<x<h(t), 0<t \leq t_{1}\right\} .
$$

Differentiating (4.18) with respect to $x$, then

$$
\partial_{t}\left(\partial_{x} u\right)-\mathcal{L}\left(\partial_{x} u\right)=0 \text { in } \mathcal{M}
$$

On the other hand, $\partial_{x} u\left(x, t_{1}\right)=0$ for any $x \in\left(x_{1}, x_{2}\right)$ in this case, and we know that $\partial_{x} u \geq 0$ by (3.26). Applying the strong maximum principle to (4.19), we obtain

$$
\partial_{x} u(x, t)=0, \quad \text { in } \mathcal{M} .
$$

So, we can define $u(x, t)=g(t)$ in $\mathcal{M}$. Considering $u(h(t), t)=K$ and $u \in C\left(\bar{\Omega}_{T}\right)$, we see that $u(x, t) \equiv K$ in $\mathcal{M}$, which contradicts that $u(x, t)<K$ for any $x<h(t)$. Therefore $h(t) \in$ $C\left[0, T_{0}\right)$.

Theorem 4.3. There exists some $t_{0} \in\left(0, T_{0}\right)$ such that $h(t)=0$ for any $t \in\left[0, t_{0}\right]$ and $h(t)$ is strictly decreasing on $\left[t_{0}, T_{0}\right)$.

Proof. Define $t_{0}=\sup \{t: t \geq 0, h(t)=0\}$. In the first, we prove that $t_{0}>0$. Otherwise, $h(0)=0$ and $h(t)<0$ for $t>0$.

Recalling the initial value, we see that

$$
\partial_{x} u(x, 0)=K e^{x} \quad \text { for any } x \in(\ln L-\ln K, 0], \quad \lim _{x \rightarrow 0^{-}} \partial_{x} u(x, 0)=K .
$$

Meanwhile, $u(x, t)=K$ in the domain $\left\{(x, t): h(t)<x<0,0<t<T_{0}\right\}$ implies that $\partial_{x} u(0, t)=$ 0 for any $t>0$ (see Figure 4 ); then $\partial_{x} u$ is not continuous at the point $(0,0)$, which contradicts $\partial_{x} u \in C\left(\overline{\Omega_{T} \backslash B_{\delta}\left(P_{0}\right)}\right)$.

In the second, we prove that $t_{0}<T_{0}$. In fact, according to Lemma 3.1, $h(t)=-\infty$ for any $t \geq T_{0}$, hence, $t_{0} \leq T_{0}$. If $t_{0}=T_{0}$, then the free boundary includes a horizontal line $t=$ $T_{0}, x \in(-\infty, 0)$. Repeating the method in the proof of Theorem 4.2, then we can obtain a contradiction. So, $t_{0}<T_{0}$. 


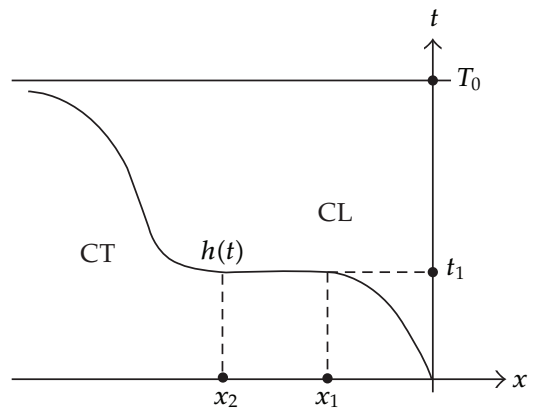

Figure 4: Discontinuous free boundary $h(t)$.

At last, we prove that $h(t)$ is strictly decreasing on $\left[t_{0}, T_{0}\right)$. Otherwise, $x=h(t)$ has a vertical part. Suppose that the vertical line is $x=x_{1}, t \in\left[t_{1}, t_{2}\right]$, then $u(x, t)=K$ for any $(x, t) \in\left(-\infty, x_{1}\right] \times\left[t_{1}, t_{2}\right]$. Since $\partial_{x} u$ is continuous across the free boundary, then $\partial_{x} u\left(x_{1}, t\right)=0$ for any $t \in\left[t_{1}, t_{2}\right]$. In this case, we infer that

$$
\partial_{t} u\left(x_{1}, t\right)=0, \quad \partial_{t}\left(\partial_{x} u\right)\left(x_{1}, t\right)=0 \quad \text { for any } t \in\left[t_{1}, t_{2}\right]
$$

On the other hand, in the domain $\mathcal{N}=\left(-\infty, x_{1}\right) \times\left(t_{1}, t_{2}\right), u$ and $\partial_{t} u$ satisfy, respectively,

$$
\begin{gathered}
\partial_{t} u-\mathcal{L} u=c \quad \text { in } \mathcal{N}, \quad u\left(x_{1}, t\right)=K \quad \text { for any } t \in\left(t_{1}, t_{2}\right), \\
\partial_{t}\left(\partial_{t} u\right)-\mathcal{L}\left(\partial_{t} u\right)=0, \quad \partial_{t} u \geq 0, \text { in } \mathcal{N}, \\
\partial_{t} u\left(x_{1}, t\right)=0 \quad \text { for any } t \in\left(t_{1}, t_{2}\right) .
\end{gathered}
$$

Then the strong maximum principle implies that $\partial_{x}\left(\partial_{t} u\right)\left(x_{1}, t\right)<0$, which contradicts the second equality in (4.22).

Theorem 4.4. $h(t)>h_{*}(t)$ for any $t \in\left[0, T_{0}\right)$ with $\lim _{t \rightarrow T_{0}^{-}} h(t)=-\infty$ (see Figure 1 ), where

$$
h_{*}(t)=\ln \frac{L}{K}+\frac{1}{\alpha} \ln \frac{(c-r L) e^{-r t}-(c-r K)}{r K}, \quad 0 \leq t<T_{0},
$$

where $\alpha$ is the positive characteristic root of $\mathcal{L} w=0$, that is, the positive root of the algebraic equation

$$
\frac{\sigma^{2}}{2} \alpha^{2}+\left(r-q-\frac{\sigma^{2}}{2}\right) \alpha-r=0
$$

Proof. Define

$$
W(x, t)=\frac{c}{r}-\left(\frac{c}{r}-L\right) e^{-r t}+\frac{K^{\alpha+1}}{L^{\alpha}} e^{\alpha x}, \quad(x, t) \in \Omega_{T_{0}} .
$$


We claim that $W(x, t) \in C^{2}\left(\bar{\Omega}_{T_{0}}\right)$ and possess the following three properties.

(i) $W(x, 0) \geq u(x, 0)$ for $-\infty<x<0$ and $W(0, t) \geq K$ for $0<t \leq T_{0}$,

(ii) $\partial_{t} W-\mathcal{L W}=c$ in $\Omega_{T_{0}}$,

(iii) $W(x, t)<K$ in $\left\{(x, t): x<h_{*}(t), 0 \leq t<T_{0}\right\}$.

In fact, if we notice that $\alpha>0$, then we have that

$$
W(x, 0)=L+\frac{K^{\alpha+1}}{L^{\alpha}} e^{\alpha x} \geq \begin{cases}L=\max \left\{L, K e^{x}\right\}=u(x, 0) & \text { if } x \leq \ln L-\ln K, \\ L+\frac{K^{\alpha+1}}{L^{\alpha}}\left(\frac{L}{K}\right)^{\alpha} \geq K \geq u(x, 0) & \text { if } \ln L-\ln K \leq x \leq 0 .\end{cases}
$$

It is obvious that $W(0, t) \geq K$. So, we obtain property (i).

Moreover, we compute

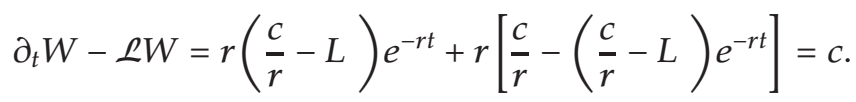

Hence, we have property (ii).

It is not difficult to check that, for any $t \in\left[0, T_{0}\right)$,

$$
W\left(h_{*}(t), t\right)=K, \quad \partial_{x} W=\frac{\alpha K^{\alpha+1}}{L^{\alpha}} e^{\alpha x}>0
$$

Then we show property (iii).

Repeating the method in the proof of Theorem 3.2, we can derive that $u \leq W$ in $\Omega_{T_{0}}$ from properties (i)-(ii). And property (iii) implies that $u<K$ in the domain $\{(x, t): x<$ $\left.h_{*}(t), 0 \leq t<T_{0}\right\}$, which means that $h(t) \geq h_{*}(t)$ for any $t \in\left[0, T_{0}\right)$.

Next, we prove that $\lim _{t \rightarrow T_{0}^{-}} h(t)=-\infty$. Otherwise, $\lim _{t \rightarrow T_{0}^{-}} h(t)=x_{1}>-\infty$; then the free boundary includes a horizontal line $t=T_{0}, x \in\left(-\infty, x_{1}\right)$. Repeating the method in the proof of Theorem 4.2, then we can obtain a contradiction. So, $\lim _{t \rightarrow T_{0}^{-}} h(t)=-\infty$.

Theorem 4.5. The free boundary $h(t) \in C^{0,1}\left[0, T_{0}\right) \cap C^{\infty}\left[t_{0}, T_{0}\right)$.

Proof. Fix $t_{1} \in\left(0, t_{0}\right)$ and $t_{2} \in\left(t_{0}, T_{0}\right)$, and denote $X=h_{*}\left(t_{2}\right)-1$. According to Theorem 4.4, the free boundary $h(t)$ while $t \in\left(t_{0}, t_{2}\right)$ lies in the domain $\mathcal{N} \triangleq\left\{(x, t): X<x<0, t_{1}<t \leq t_{2}\right\}$ (see Figure 5).

In the first, we prove that there exists an $M_{0}>0$ such that

$$
M_{0} \partial_{x} u-\partial_{t} u \geq 0 \text { in } \mathcal{N}
$$

In fact, $u, \partial_{t} u$ satisfy the equations

$$
\partial_{t} u-\mathcal{L} u=c, \quad \partial_{t}\left(\partial_{t} u\right)-\mathcal{L}\left(\partial_{t} u\right)=0, \quad(x, t) \in \mathrm{CT},
$$




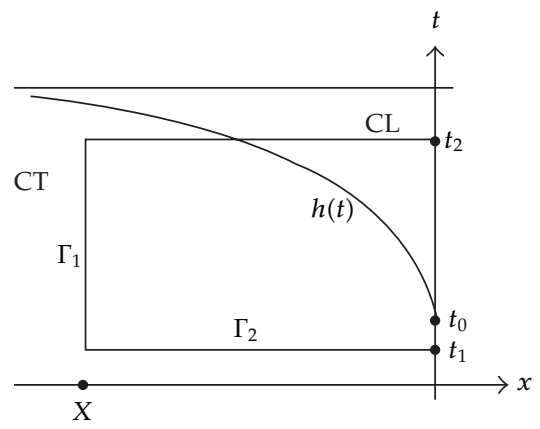

Figure 5: The free boundary $h(t)$.

then the interior estimate of the parabolic equation implies that there exists a positive constant $C$ such that

$$
\partial_{t} u(x, t) \leq C \quad \text { on } \Gamma_{1} \cup \Gamma_{2},
$$

here

$$
\Gamma_{1} \triangleq\left\{x=X, t_{1} \leq t \leq t_{2}\right\}, \quad \Gamma_{2} \triangleq\left\{X \leq x \leq 0, t=t_{1}\right\}
$$

On the other hand, we see that $\partial_{t} u \geq 0$ in $\bar{\Omega}_{T}$ from (3.27), and $\partial_{t} u(0, t)=0$. Applying the strong maximum principle to $\partial_{t} u(x, t)$, we deduce that

$$
\partial_{t x} u(0, t)<0, \quad t \in\left(0, t_{0}\right)
$$

It means that $\partial_{x} u(0, t)$ is strictly decreasing on $\left[0, t_{0}\right]$. It follows that, by $\partial_{x} u\left(0, t_{0}\right)=0$,

$$
\partial_{x} u\left(0, t_{1}\right)>0
$$

Moreover,

$$
\partial_{x} u \geq 0, \quad \partial_{t}\left(\partial_{x} u\right)-\mathcal{L}\left(\partial_{x} u\right)=0, \quad(x, t) \in \mathrm{CT} .
$$

Employing the strong maximum principle, we see that there is a $\delta>0$, such that

$$
\partial_{x} u(x, t) \geq \delta \quad \text { on } \Gamma_{1} \cup \Gamma_{2},
$$

Provided that $\delta$ is small enough. Combining (4.32), there exists a positive $M_{0}=(C / \delta)+1$ such that

$$
M_{0} \partial_{x} u-\partial_{t} u \geq \delta \quad \text { on } \Gamma_{1} \cup \Gamma_{2}
$$




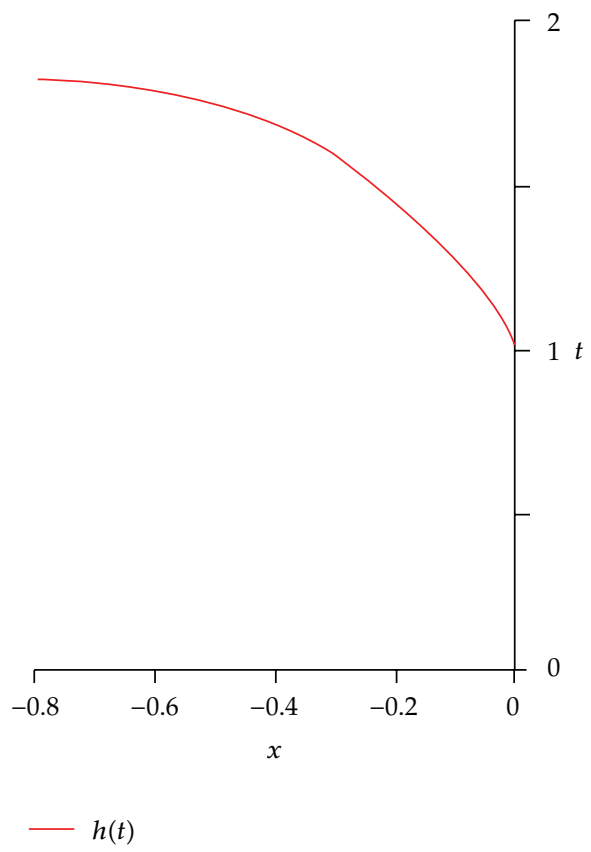

Figure 6: The free boundary.

Next, we concentrate on problem (1.4) in the domain $\mathcal{N}$. It is clear that $u$ satisfies

$$
\begin{gathered}
\partial_{t} u-\mathcal{L} u=c, \quad \text { if } u<K, \quad(x, t) \in \mathcal{N}, \\
\partial_{t} u-\mathcal{L} u \leq c, \quad \text { if } u=K, \quad(x, t) \in \mathcal{N}, \\
u(X, t)=u(X, t), \quad u(0, t)=K, \quad t_{1} \leq t \leq t_{2}, \\
u\left(x, t_{1}\right)=u\left(x, t_{1}\right), \quad X \leq x \leq 0 .
\end{gathered}
$$

And we can use the following problem to approximate the above problem:

$$
\begin{gathered}
\partial_{t} u_{\varepsilon}-\mathcal{L} u_{\varepsilon}+\beta_{\varepsilon}\left(u_{\varepsilon}-K\right)=c, \quad \text { in } \mathcal{N}, \\
u_{\varepsilon}(X, t)=u(X, t), \quad u_{\varepsilon}(0, t)=K, \quad t_{1} \leq t \leq t_{2}, \\
u_{\varepsilon}\left(x, t_{1}\right)=u\left(x, t_{1}\right), \quad X \leq x \leq 0 .
\end{gathered}
$$

Recalling (4.38), we see that, if $\varepsilon$ is small enough, $M_{0} \partial_{x} u_{\varepsilon}-\partial_{t} u_{\varepsilon} \geq 0$ on the parabolic boundary of $\mathcal{N}$. Moreover, $w \triangleq M_{0} \partial_{x} u_{\varepsilon}-\partial_{t} u_{\varepsilon}$ satisfies

$$
\partial_{t} w-\mathcal{L} w+\beta_{\varepsilon}^{\prime}\left(u_{\varepsilon}-K\right) w=0 .
$$


Applying the comparison principle, we obtain

$$
M_{0} \partial_{x} u_{\varepsilon}-\partial_{t} u_{\varepsilon}=w \geq 0 \text { in } \mathcal{N}
$$

As the method in the proof of Theorem 3.3, we can show that $u_{\varepsilon}$ weakly converges to $u$ in $W_{p}^{2,1}(\mathcal{N})$ and (4.30) is obvious.

On the other hand, we see that $M \partial_{x} u+\partial_{t} u \geq 0$ in $\mathcal{N}$ for any positive number $M$ from (3.26) and (3.27). So,

$$
M_{0} \partial_{x} u \pm \partial_{t} u \geq 0 \text { in } \mathcal{N},
$$

which means that there exists a uniform cone such that the free boundary should lies in the cone. As the method in [9], it is easy to derive that $h(t) \in C^{0,1}\left[t_{1}, t_{2}\right]$. Moreover $h(t) \in$ $C^{\infty}\left[t_{0}, t_{2}\right]$ can be deduced by the bootstrap method. Since $t_{2}$ is arbitrary and the free boundary is a vertical line while $t \in\left(0, t_{0}\right)$, then $h(t) \in C^{0,1}\left[0, T_{0}\right) \cap C^{\infty}\left[t_{0}, T_{0}\right)$.

\section{Numerical Results}

Applying the binomial tree method to problem (1.4), we achieve the following numerical results-Figure 6:

Plot of the optimal exercise boundary $h(t)$ is a function of $t$. The parameter values used in the calculations are $r=0.2, q=0.1, \sigma=0.3, L=1, K=1.5, c=0.5, T=2$, and $n=3000$. In this case, the free boundary is increasing with $x(0)=0$. The numerical result is coincided with that of our proof (see Figure 6).

\section{Acknowledgments}

The project is supported by NNSF of China (nos. 10971073, 11071085, and 10901060) and NNSF of Guang Dong province (no. 9451063101002091).

\section{References}

[1] M. Sîrbu, I. Pikovsky, and S. E. Shreve, "Perpetual convertible bonds," SIAM Journal on Control and Optimization, vol. 43, no. 1, pp. 58-85, 2004.

[2] M. Sîrbu and S. E. Shreve, "A two-person game for pricing convertible bonds," SIAM Journal on Control and Optimization, vol. 45, no. 4, pp. 1508-1539, 2006.

[3] P. Wilmott, Derivatives, the Theory and Practice of Financial Engineering, John Wiley \& Sons, New York, NY, USA, 1998.

[4] Y. Kifer, "Game options," Finance and Stochastics, vol. 4, no. 4, pp. 443-463, 2000.

[5] Y. Kifer, "Error estimates for binomial approximations of game options," The Annals of Applied Probability, vol. 16, no. 2, pp. 984-1033, 2006.

[6] C. Kühn and A. E. Kyprianou, “Callable puts as composite exotic options,” Mathematical Finance, vol. 17, no. 4, pp. 487-502, 2007.

[7] A. E. Kyprianou, "Some calculations for Israeli options," Finance and Stochastics, vol. 8, no. 1, pp. 73-86, 2004.

[8] F. Black and M. Scholes, "The pricing of options and coperate liabilities," Journal of Political Economy, vol. 81, pp. 637-659, 1973. 
[9] Z. Yang and F. Yi, "A free boundary problem arising from pricing convertible bond," Applicable Analysis, vol. 89, no. 3, pp. 307-323, 2010.

[10] A. Friedman, "Parabolic variational inequalities in one space dimension and smoothness of the free boundary," Journal of Functional Analysis, vol. 18, pp. 151-176, 1975.

[11] A. Blanchet, "On the regularity of the free boundary in the parabolic obstacle problem. Application to American options," Nonlinear Analysis: Theory, Methods \& Applications, vol. 65, no. 7, pp. 1362-1378, 2006.

[12] A. Blanchet, J. Dolbeault, and R. Monneau, "On the continuity of the time derivative of the solution to the parabolic obstacle problem with variable coefficients," Journal de Mathématiques Pures et Appliquées. Neuvième Série, vol. 85, no. 3, pp. 371-414, 2006.

[13] L. Caffarelli, A. Petrosyan, and H. Shahgholian, "Regularity of a free boundary in parabolic potential theory," Journal of the American Mathematical Society, vol. 17, no. 4, pp. 827-869, 2004.

[14] A. Petrosyan and H. Shahgholian, "Parabolic obstacle problems applied to finance," in Recent Developments in Nonlinear Partial Differential Equations, vol. 439 of Contemporary Mathematics, pp. 117133, American Mathematical Society, Providence, RI, USA, 2007.

[15] A. Friedman, "Stochastic games and variational inequalities," Archive for Rational Mechanics and Analysis, vol. 51, pp. 321-346, 1973.

[16] A. Friedman, Variational Principles and Free-Boundary Problems, Pure and Applied Mathematics, John Wiley \& Sons, New York, NY, USA, 1982.

[17] D. Gilbarg and N. S. Trudinger, Elliptic Partial Differential Equations of Second Order, vol. 224 of Grundlehren der Mathematischen Wissenschaften, Springer, Berlin, Germany, 2nd edition, 1983.

[18] O. A. Ladyženskaja, V. A. Solonnikov, and N. N. Uralceva, Linear and Quasi-linear Equations of Parabolic Type, American Mathematical Society, Providence, RI, USA, 1968.

[19] F. Yi, Z. Yang, and X. Wang, "A variational inequality arising from European installment call options pricing," SIAM Journal on Mathematical Analysis, vol. 40, no. 1, pp. 306-326, 2008.

[20] K. Tso, "On Aleksandrov, Bakel'man type maximum principle for second order parabolic equations," Communications in Partial Differential Equations, vol. 10, no. 5, pp. 543-553, 1985. 